Artículos Científicos

\title{
Instrumento certificador de tecnologías de la información y comunicación y tecnologías del aprendizaje y el conocimiento para docentes universitarios
}

\begin{abstract}
Instrument Certifier of Information and Communication Technologies and Learning and Knowledge Technologies for University Teachers
\end{abstract}

Instrumento de certificação de tecnologias de informação e comunicação e tecnologias de aprendizagem e conhecimento para professores universitários

Elvira Ivone González Jaimes

Universidad Autónoma del Estado de México, Estado de México, México ivonegj@hotmail.com http://orcid.org/0000-0002-5328-5586

Asdrubal López Chau

Universidad Autónoma del Estado de México, Estado de México, México

alchau@uaemex.mx https://orcid.org/0000-0001-5254-0939

Valentín Trujillo Mora

Universidad Autónoma del Estado de México, Estado de México, México

vtrujillom@uaemex.mx https://orcid.org/0000-0002-5936-4795

Jorge Bautista López

Universidad Autónoma del Estado de México, Estado de México, México

jbautistal@uaemex.mx https://orcid.org/0000-0002-0055-2310 


\section{Resumen}

Este artículo presenta los resultados de la investigación cuyo objetivo consistió en la elaboración de un instrumento innovador, ágil, veraz y confiable para evaluar y certificar al personal académico universitario. Un instrumento capaz de detectar cuatro niveles de conocimientos y uso en tecnologías de la información y la comunicación (TIC) y tecnologías del aprendizaje y el conocimiento (TAC).

Se trató de un estudio cuasiexperimental dividido en tres fases: a) elaboración de la prueba con diseño mixto transformativo secuencial; $b$ ) evaluación de la prueba con diseño mixto secuencial, y c) sistematización con App Ionic 3.

Una vez realizados los análisis cualitativos y cuantitativos, el instrumento resultó versátil y transferible en 1) selección y contratación de personal, 2) identificar el nivel conocimientos que tiene el personal, 3) crear programas y/o cursos que refuerce o cree nuevas habilidades en TIC y TAC, 4) categorizar al personal de acuerdo su nivel de conocimientos, 5) promocionar al personal para adquirir beneficios o prestaciones laborales, 6) evidenciar el nivel que tiene los académicos en la evaluación que realizan los organismos acreditadores y 7) constancia curricular de posesión de nivel de conocimiento y uso en TIC y TAC en área académica.

Dicha prueba puede ser aplicable de forma individual o colectiva y obtener calificaciones a través de un método manual, por lo que se anexa una guía de usuario, o electrónico, para lo cual se requiere solicitar la página web a los autores.

Palabras claves: evaluación del docente; tecnologías educativas, tecnologías de la información y comunicación.

\section{Abstract}

The objective of this study was the development of an innovative, agile, truthful and reliable instrument to evaluate and certify university academic staff; a detector of four levels of knowledge and use in information and communication technologies (ICT) and learning and knowledge technologies (LKT).

This investigation was divided in three phases: a) Elaboration of the test with sequential transformative mixed design. b) Evaluation of the test with sequential mixed design. c) Systematized with App Ionic 3.

After the qualitative and quantitative analyses, the instrument turned out to be versatile, transferable in: 1) Selection and hiring of personnel, 2) Identify the level of 
knowledge that the personnel have, 3) Create programs and/or courses that reinforce or create new skills in ICT and TAC, 4) Categorize personnel according to their level of knowledge, 5) Promote personnel to acquire benefits or labor benefits, 6) Evidence the level that the academics have in the evaluation that the accrediting bodies carry out and 7) Curricular evidence of possession of the level of knowledge and use in ICT and LKT in the academic area.

This this is applicable in individual or collective form and the qualification could be manual, reason why it is annexed instructions of user, or electronic - it is required to request the web page to the authors.

Keywords: teacher evaluation; educational technologies, information technologies.

\section{Resumo}

Este artigo apresenta os resultados da pesquisa cujo objetivo foi o desenvolvimento de um instrumento inovador, ágil, verdadeiro e confiável para avaliar e certificar o corpo docente universitário. Um instrumento capaz de detectar quatro níveis de conhecimento e uso em tecnologias de informação e comunicação (TIC) e tecnologias de aprendizagem e conhecimento (TAC).

Trata-se de um estudo quase-experimental, dividido em três fases: a) elaboração do teste com desenho misto transformacional sequencial; b) avaliação do teste com desenho misto sequencial ec) sistematização com App Ionic 3.

Uma vez que as análises qualitativas e quantitativas foram realizadas, o instrumento foi versátil e transferível em 1) recrutamento e recrutamento, 2) identificar o nível de conhecimento que a equipe possui, 3) criar programas e / ou cursos que reforçam ou criam novas habilidades em TIC e TAC, 4) categorizam o pessoal de acordo com o seu nível de conhecimento, 5) promovem o pessoal para obter benefícios ou benefícios do trabalho, 6) mostram o nível de académicos na avaliação realizada pelos organismos de acreditação e 7) evidência do currículo de posse de nível de conhecimento e uso em TIC e TAC na área acadêmica.

Esse teste pode ser aplicado individual ou coletivamente e obter qualificações por meio de um método manual, portanto, um guia do usuário, ou eletrônico, é anexado, para o qual é necessário solicitar o site aos autores.

Palavras-chave: avaliação de professores; tecnologias educacionais, tecnologias de informação e comunicação. 


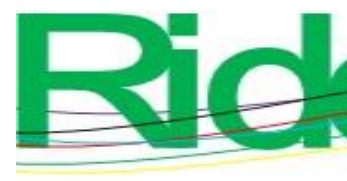

Fecha Recepción: Febrero 2019
Revista Iberoamericana para la

Investigación y el Desarrollo Educativo

ISSN $2007-7467$

Fecha Aceptación: Junio 2019

\section{Introducción}

La presente investigación elabora, valida, sistematiza y estandariza un instrumento para evaluar al personal académico universitario en cuanto al conocimiento y uso en tecnologías de la información y la comunicación (TIC) y tecnologías del aprendizaje y el conocimiento (TAC). Es un instrumento cuidadosamente elaborado, capaz de estimar cuatro niveles de conocimiento y uso en tecnología aplicada en el proceso de enseñanza-aprendizaje impartido por académicos.

Entre los principales criterios evaluados por los organismos acreditadores se encuentran los programas educativos y la implementación de estos. En la implementación destaca el conocimiento y el uso de las TIC y TAC del personal académico, ya que son herramientas esenciales para la capacitación integral de los estudiantes: impulsan habilidades tecnológicas indispensables para ingresar al mercado laboral.

El instrumento presentado es un producto de medición innovador, ágil, veraz y confiable. Es capaz de detectar niveles de conocimientos y uso en TIC y TAC que posee el personal académico universitario de acuerdo con los siguientes parámetros: $a$ ) conceptos teóricos ratificados en otras investigaciones certificadoras reconocidas, $b$ ) pilotaje de expertos y c) criterios establecidos por el Consejo para la Acreditación de la Educación Superior (Copaes). Lo que permite al instrumento ser certificador de conocimientos y uso en TIC y TAC.

Por lo que los resultados de la evaluación del instrumento sirven como evidencia, elemento requerido por los organismos acreditadores, y agilizan los procesos de medir la calidad educativa y su pertinencia en la educación superior.

Ahora bien, la aplicabilidad del instrumento es aceptable porque recopila datos/información de forma sistematizada y permite obtener resultados individuales o de grupo. Asimismo, presenta una adecuada transferencia en universidades de nivel superior mexicanas porque está elaborado bajo los criterios que solicita el Copaes y estandarizado con una población universitaria de 20 estados de México.

Los organismos acreditadores registrados en el Copaes solicitan evidencias de evaluaciones siguiendo los siguiente criterios:

a) Indicadores imprescindibles, apartados: 4. Plan de Estudios, inciso 4.2. Cumpliendo con la enseñanza de tecnología. 5. Personal Académico, inciso 5.1. Formación académica y experiencia docente (enseñanza con uso de la tecnología). 


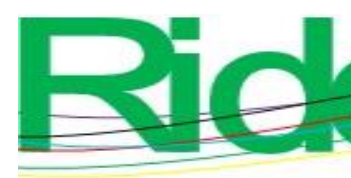

Revista Iberoamericana para la

Investigación y el Desarrollo Educativo

ISSN $2007-7467$

b) Indicadores indispensable, apartados: 4. Plan de Estudios, inciso 4.3. Respuesta a las necesidades de la disciplina, sus tendencias y áreas de aplicación (área de conocimiento tecnologías). 5. Personal Académico, incisos 5.6. Habilidades docentes (tecnológicas) y 5.7. Método de enseñanza (uso de herramientas y plataformas digitales).

c) Indicadores necesarios, apartado 3. Gestión administrativa y financiera en el apartado, inciso 3.4. Uso de centros de cómputo, aulas y/o laboratorios digitales.

\section{¿Quiénes regulan y validan la calidad educativa?}

Los organismos acreditadores internacionales y nacionales son los que validan y certifican la calidad educativa con base en la cultura, ciencia y economía mundial.

Las evaluaciones se han vuelto necesarias a nivel internacional porque existen organizaciones que determinan continuamente la calidad educativa. La Organización de las Naciones Unidas para la Educación, la Ciencia y la Cultura (Unesco) es una de ellas. En el documento Educación hacia 2030, en su indicador mundial 4.4, se menciona lo siguiente: "De aquí a 2030, aumentar considerablemente el número de jóvenes y adultos con competencias necesarias, en particular técnicas y profesionales, para acceder al empleo, el trabajo decente y el emprendimiento" (Unesco, 2016, p. 78).

Desde hace décadas se mide la calidad educativa y su pertinencia para el empleo. Por ejemplo, el Tuning Project (vocablos en inglés que significan 'proyecto de sincronización'), concebido por universidades europeas y presentado en 2003, tiene el objetivo de medir, comparar y regular el aprendizaje en términos de competencias genéricas y específicas. Creado con un lenguaje universal de conocimientos y destrezas, utilizado en diferentes áreas disciplinarias, ha sido la base del procedimiento de homologación de programas de estudios entre universidades de diversos países europeos, y ha posibilitado a los egresados laborar en otras localidades de la Unión Europea (González y Wagenaar, 2003). En América Latina, por su parte, existe una adaptación de dicha iniciativa: Alfa Tuning América Latina, la cual también está basada en la transferencia de conocimientos, homologación de programas de estudio universitario y el desarrollo social y económico de la zona (Beneitone et al., 2007).

En México se realizan encuestas nacionales para conocer las perspectivas de la política educativa dirigidas por la Organización de Cooperación de Desarrollo Económico (OCDE). Dichas encuestas evalúan los siguientes factores: $a$ ) Estudiantes en cuanto a equidad y calidad educativa, índices para inclusión en el futuro beneficioso y remunerado que requiere de conocimiento en competencias y habilidades tecnológicas; b) Instituciones educativas en cuanto a la evaluación del control de calidad de la enseñanza de habilidades tecnológicas 


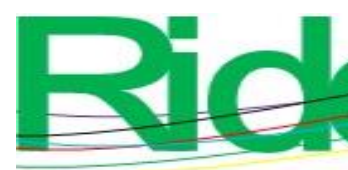

Revista Iberoamericana para la

Investigación y el Desarrollo Educativo

ISSN $2007-7467$

como requerimientos para el ingreso al mercado laboral, y c) Sistemas educativos para llevar a cabo la política educativa en términos de gobernabilidad acorde al crecimiento mundial socioeconómico (OCDE, 2017).

De acuerdo con los índices arrojados por la encuesta del 2016-2017, la OCDE (2018) aconseja a las autoridades mexicanas lo puesto a continuación:

Aumentar las sinergias entre la Secretaría de Hacienda, Crédito Público, la Secretaría de Economía, la Secretaría de Hacienda, la Secretaría de Trabajo y el Consejo Nacional de Ciencia y Tecnología para que mejorar la calidad y pertinencia de la educación para las necesidades de desarrollo a corto y largo plazo del país (p. 17).

Otra institución como Organización Internacional del Trabajo recomienda líneas de acción a la SEP y otras instituciones nacionales educativas para regular y coordinar la educación acorde al desarrollo productivo y el mercado laboral nacional e internacional.

\section{El proceso de certificación en México y sus actores}

En los párrafos anteriores se observa cómo las organizaciones de tipo internacional dictan recomendaciones para las instituciones educativas mexicanas. La ejecución de las recomendaciones regulan los estándares de calidad educativa y su pertinencia con los índices de producción y necesidades del mercado laboral nacional e internacional.

La Secretaría de Educación Pública [SEP] es la encargada de orientar y medir estas líneas de acción estipuladas por las diferentes organizaciones internacionales. Y como es sabido, la SEP dirige al Copaes, única instancia autorizada por el Gobierno Federal para acreditar programas educativos de nivel superior en México (Copaes, 2018).

Otra instancia que apoya y supervisa los procesos de certificación es la Asociación Nacional de Universidades e Instituciones de Educación Superior (Anuies).

El Copaes, cuyo objetivo, como ya se mencionó, es evaluar la calidad educativa, realiza su labor a partir de los siguientes lineamientos: 1) La equidad e imparcialidad, 2) Congruencia y confiabilidad, 3) Control y aseguramiento de la calidad, 4) Responsabilidad y seriedad y 5) Transparencia y rendición de cuentas (Copaes, 2018).

El Copaes posee el registro de todos organismos acreditadores autorizados, los cuales son ajenos a cualquier interés particular de persona, grupo o institución (Copaes, 2016).

El primer organismo acreditador en México es el llamado Comités Interinstitucionales para la Evaluación de la Educación Superior (CIEES). Este es capaz de medir y avalar a la institución o programa de educación superior gracias a que cuenta con nueve comités 


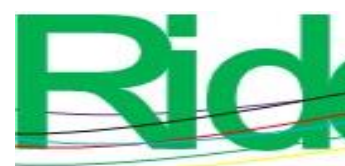

Revista Iberoamericana para la

Investigación y el Desarrollo Educativo

ISSN $2007-7467$

interinstitucionales, acordes a las diferentes áreas de conocimiento, midiendo y certificando en Nivel I.

El Nivel II de calidad educativa es más específico y es evaluado por organismos certificadores especializados en diferentes áreas de conocimiento; todas las evaluaciones son reportadas y registradas en Copaes (2018) por medio de categorías.

Las categorías contienen un conjunto de criterios, indicadores y estándares, los cuales son sujetos a evaluaciones detalladas para poder emitir un dictamen de acreditación, según el Copaes (2016); y son: 1) Personal académico, 2) Estudiantes, 3) Plan de estudios, 4) Evaluación del aprendizaje, 5) Formación integral, 6) Servicios de apoyo al aprendizaje, 7) Vinculación, Extensión, 8) Investigación, 9) Infraestructura y equipamiento y 10) Gestión administrativa y financiera. Aunado a esto, cada categoría tiene subcategorías que engloban la forma de evaluación específica (Copaes, 2016).

El presente estudio se focalizó en la categoría 1) Personal académico. En ella se encuentra las subcategorías:

a) Reclutamiento, selección y contratación: son los procesos para el ingreso a la institución académica, que considera la contratación de personal que tenga las aptitudes y habilidades para el manejo de las TIC y TAC.

b) Desarrollo: proceso que mide la trayectoria (como la asignación de programas y/o cursos que refuercen o creen nuevas habilidades, incluyendo el uso de TIC y TAC como competencia transversal).

c) Categorización y nivel de estudios: procesos que evalúan el equilibrio del personal académico en las actividades que debe de realizar dentro de la institución y sus competencias, donde se puede retribuir el conocimiento y uso de TIC y TAC

d) Evaluación interna: estimación del rendimiento de los académicos y la promoción que han recibido de acuerdo con su desempeño (Consejo Nacional para la Enseñanza e Investigación en Psicología [CNEIP], 2018b, pp. 17-20).

Esta misma tendencia se ve reflejada en las instituciones de educación superior, tal y como se observa en la Universidad Autónoma del Estado de México (UAEM).

El fin primordial de los estudios profesionales es la formación del estudiante, mediante el desarrollo de sus facultades, aptitudes, actitudes y valores, para ejercer actividades profesionales de alta calidad y evolucionar en el campo laboral, así como para desempeñarse en la indagación, creación y recreación del conocimiento" Reglamento de Estudios Profesionales (UAEM, 2017, p. 195). 


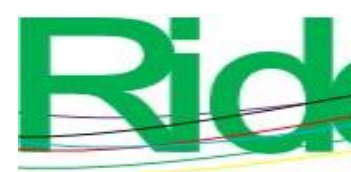

Revista Iberoamericana para la

Investigación y el Desarrollo Educativo

ISSN $2007-7467$

Cabe señalar que esta institución posee $92 \%$ de sus programas educativos con alguna acreditación de diversos organismos. Por su trayectoria, la UAEM fue seleccionada y se invitó a sus docentes expertos en TIC y TAC a participar en esta investigación, así como a los académicos expertos necesarios para poder realizar el análisis cualitativo.

\section{Evaluación de las TIC y TAC en educación superior}

Las instituciones de educación superior han elaborado procedimientos e instrumentos de medición porque requieren evidencias que comprueben su calidad educativa. A través de ellos se evalúa el criterio de conocimiento y el uso de las TIC y TAC. Aunque estas se relacionan con competencias instrumentales transversales del académico (Prendes, 2010).

Los índices y evidencias solicitadas por los organismos acreditadores en cuanto a los rubros de evaluación al personal académico universitario son los lineamientos generales de tipo personal (curriculares). En la trayectoria del académico es posible observar parámetros específicos de procesos de enseñanza-aprendizaje, ajustados a los programas de aprendizaje y el desarrollo, la actualización de competencias específicas y transversales en el uso de las TIC (Cabero y Gutiérrez, 2015). También se menciona requisiciones para el personal académico.

Conlleva tres dimensiones relevantes en la consideración de la formación por competencias: a) el perfil profesional como referente del diseño curricular, $b$ ) el espacio formativo, integrando la institución formativa y la institución sociolaboral y c) el tiempo formativo, que se proyecta a lo largo de toda la vida, siendo insuficiente la formación inicial y resaltando el protagonismo de la formación continua (Tejada y Ruiz, 2016, p. 7).

\section{Instrumentos para evaluar las TIC y TAC en nivel superior}

Bajo el auspicio de la Comisión Europea se han realizado investigaciones sobre las TIC y se han diseñado varios instrumentos en la Web, como es DigComp, que mide las competencias digitales en una población común (Ferrari, 2013). Este, además, fue fundamento para la construcción del instrumento DigCompEdu, que también mide competencias digitales en profesores de cualquier nivel académico y es respaldado por el Centro Común de Investigación (JRC, por sus siglas en inglés). Ambos instrumentos están contemplados en la Estrategia Europa 2020, agenda que evalúa el hoy de la enseñanza acorde al empleo del mañana (JRC, 2017). 


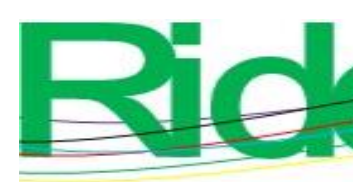

Revista Iberoamericana para la

Investigación y el Desarrollo Educativo

ISSN $2007-7467$

Bajo el patrocinio del Ministerio de Educación Español y el apoyo del Grupo de Investigación de Tecnología Educativa de la Universidad de Murcia, se construyó en 2010 el cuestionario titulado "Competencias TIC para la docencia en la Universidad Pública Española" (Prendes, 2010), el cual sirvió como antecedente para construir el instrumento para certificar la competencia en TIC de Durán (2015) y Durán, Gutiérrez y Prendes (2016). Los preliminares de este último consisten en lo siguiente: 1) posee validación interna (grupo de discusión) apoyado en las buenas prácticas del profesorado (Prendes y Gutiérrez, 2013) y 2) posee validación externa con prueba piloto en profesores universitarios y con parámetros de la Unión Europea (Gutiérrez 2011 y 2014). Este tipo de evaluaciones continuas sirven como base para los organismos certificadores en España.

En el contexto latinoamericano, Taquez, Rengifo y Mejía (2017), investigadores de la Escuela de Ciencias de la Educación de la Universidad Icesi de Cali, Colombia, construyeron un instrumento denominado SABER-TIC, el cual permite obtener información del conocimiento y apropiación de las TIC en los profesores, con el objetivo de identificar las "necesidades educativas para el diseño de planes de formación docente para la integración de las TIC en los procesos de enseñanza y aprendizaje” (Taquez et al., 2017, párr. 9).

Como se ha visto, existen diferentes instrumentos que mide las TIC. Pues es un área esencial en la educación, por lo que existen especialistas en describir el impacto que tiene las TIC y las TAC en la educación universitaria (Ramírez y Casillas, 2014), consideradas aquí herramientas indispensables para la ejecución de la educación integral.

\section{Bases para la construcción del instrumento propuesto en esta investigación}

El instrumento propuesto aquí mide las TIC y TAC. Se obtuvo la validez de constructo a través de las partes teóricas comprobadas que sostienen los dos instrumentos anteriormente mencionados: 1) El propuesto por Durán (2015) y Durán et al. (2016), el cual sostiene y verifica los aspectos de evaluación transversal y de dominio que requiere la enseñanzaaprendizaje con TIC. Lo anterior a través de la medición de tres bloques intrínsecos de la competencia TIC y tres niveles de dominio, lo que da el rango de certificación (Durán, 2015; Durán et al., 2016) y 2) el instrumento SABER-TIC, que fundamenta y mide la parte de uso y apropiación de las TIC, incluyendo en la práctica a estas herramientas, ya que están en la implementación de la tecnología en el aprendizaje (Taquez et al., 2017).

El instrumento es útil porque permite evaluar y certificar de forma ágil, válida y confiable al personal académico en a) nivel de conocimientos en TIC y TAC dentro del proceso de selección de personal, $b$ ) detectar las necesidades de capacitación en TIC y TAC, 


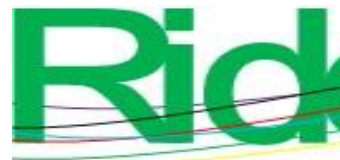

Revista Iberoamericana para la

Investigación y el Desarrollo Educativo

ISSN $2007-7467$

c) detectar el nivel conocimientos en TIC y TAC para promociones de categorización y $d$ ) elaborar constancias de acreditación en cuatro niveles de conocimientos en TIC y TAC; evidencias válidas y confiables para cuando son evaluados por organismos certificadores externos.

\section{Método}

Se trata de estudio cuasiexperimental desarrollado en tres fases, a saber: 1) Elaboración del instrumento: se implementó un diseño transformativo secuencial (Ditras); 2) Evaluación del instrumento provisional: se utilizó un diseño mixto secuencial [Dimixs] (Llobel, Pérez y Navarro, 1996), y 3) Alineación instrumento definitivo: se sistematizó y estandarización (ver figura 1).

Figura 1. Estudio cuasiexperimental con diseños mixtos

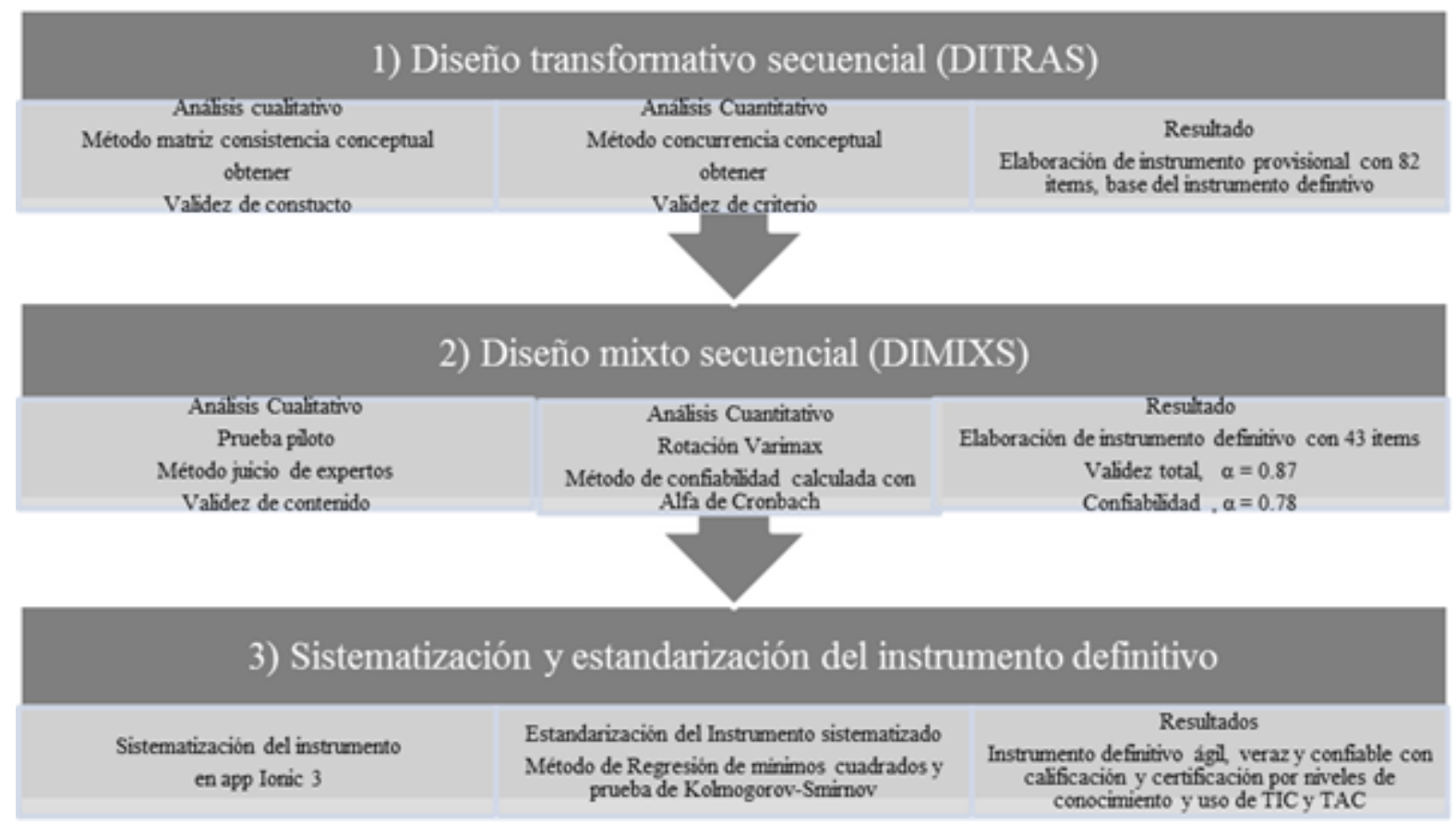

Fuente: Elaboración propia 


\section{Procedimiento}

Fase 1. Elaboración del instrumento.

Nivel 1.1. Se aplicó un análisis cualitativo a través de una matriz de consistencia conceptual para obtener la validez de constructo. Ahí se compararon los ítems elaborados con teorías reconocidas y probadas en dos instrumentos realizados por Durán (2015) y Durán et al. (2016) y Taquez et al. (2017).

Nivel 1.2. Se aplicó un análisis cuantitativo a través del método concurrencia conceptual para obtener la validez de criterio. En este punto se obtuvo la frecuencia de los estándares (resultado de la práctica del académico), emitidos por la Clasificación Mexicana de Programas de Estudio por Campos de Formación Académica, editados por el Instituto Nacional de Estadística y Geografía [Inegi] (2012) y respaldados por el Copaes y la SEP.

Por la cantidad de la información se seleccionó al azar los estándares de tres áreas conocimiento específico: a) Área de negocios y administrativas: Consejo de Acreditación de la Enseñanza en Contaduría y Administración [Caceca] (2018); b) Área ingenierías: Consejo Nacional de Acreditación en Informática y Computación [Conaic] (2017), y c) Área de ciencias sociales: Consejo Nacional para la Enseñanza e Investigación en Psicología [CNEIP] (2018a).

Nivel 1.3. Elaboración de un instrumento provisional con 82 ítems integrados y transformados.

Fase 2. Evaluación del instrumento provisional.

Nivel 2.1. Se utilizó un análisis cualitativo en la prueba piloto a través del método de juicio de expertos para obtener la validez de contenido; participaron 36 académicos expertos en tres áreas conocimiento; emitieron su criterio sobre el conocimiento y uso de las TIC y TAC; calificaron uno (conocimiento), dos (uso personal) y tres (uso académico). Los ítems podían ser calificados con los tres puntos o dejarse de calificar, para así poder obtener los criterios con mayor puntuación de cada ítem analizado.

Nivel 2.2. Con los puntajes se realizó un análisis cuantitativo factorial exploratorio a través del método extracción de componentes principales, con rotación varimax para simplificar la interpretación de los factores en las diferentes partes. Y se aplicó el índice de adecuación muestral de Kaiser-Meyer-Olkin (KMO) $\geq 0.5$ (Kerlinger, 2009).

Después de extraer a los ítems principales por tener elevadas correlaciones, $r>0.05$, se aplicó la prueba de confiabilidad: se utilizó la prueba de alfa de Cronbach > 0.75 (Cronbach, 1998). 


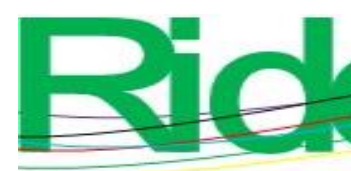

Revista Iberoamericana para la

Investigación y el Desarrollo Educativo

ISSN $2007-7467$

Nivel 2.3. Elaboración del instrumento definitivo.

El instrumento definitivo contiene 43 ítems. Fue dividido en tres partes: 1) Consideraciones generales (cinco reactivos), 2) actividades académicas (15 reactivos) y 3) Uso de herramientas educativas (23 reactivos).

Fase 3. Sistematización y estandarización del instrumento definitivo.

Nivel 3.1. El instrumento fue sistematizado a través de aplicación informática Ionic 3. Las iónicas fueron creadas y desarrolladas en línea con comandos de Ionic (CLI) y utilizan Córdova como aplicación nativa.

En este nivel también se personalizó la paleta de colores, logotipos, mensajes de bienvenida y despedida. La interfaz del diseño de preguntas es para modalidad intuitiva y con tres secciones principales: el texto (parte superior), tipo de pregunta (centro) y opciones (inferior).

Además, esta herramienta almacena base de datos en formato HTML. Y es capaz de transportar datos a formatos XLS, XLSX, CSV, JSON y PDF.

Cabe aclarar que Ionic 3 no requiere de una infraestructura dedicada, así como la instalación y configuración de servidores dedicados o software. Puede ser utilizada en móviles, lo que ofrece mayor versatilidad de uso.

Nivel 3.2. Estandarización de la app Ionic 3. Fue probada con éxito con 338 académicos universitarios, lo que ayudó a verificar el proceso de captura y almacenamiento. Los académicos eran de 20 estados de México, lo que, a su vez, ayudó a obtener la validación externa; se seleccionaron semejantes resultados a través de la prueba de regresión de mínimos cuadrados para observar la variabilidad entre los grupos considerando a $r 2>0.75$ en $p<0.05$. Por último, se evaluó la distribución de la población a través de prueba Kolmogorov-Smirnov, donde se observó semejanza a la distribución normal, lo que garantiza la estandarización en la población docente mexicana.

Nivel 3.3. Exposición de un instrumento definitivo ágil, veraz y confiable con calificación y certificación por niveles de conocimiento y uso de TIC y TAC

Se conservó su forma, validez y confiabilidad, y se corroboró lo descrito por Kerlinger en 2009, quien menciona que los instrumentos de evaluación deben de mantener las características para lo que fueron diseñados, sin importar los procesos de implementación. 


\section{Resultados}

El instrumento fue elaborado minuciosamente. Primero, se revisó la validez de constructo a través de referencias de teóricos y test previos (Gutiérrez y Prendes 2016; Taquez, et al., 2017). La validez del contenido se revisó asimismo con base en el Copaes y la SEP (Inegi, 2012).

En cuanto a la validez interna, realizada con los 36 personal académico, se obtuvo $k=$ 0.72; para la varianza de los resultados se empleó la prueba de Kaiser-Meyer-Olkin (coeficiente $=0.789)$ y de la de esfericidad de Bartlett $\left(\chi^{2}=964.06, p=.001\right)$. Por considerarse un instrumento unidimensional, se aplicó el método de extracción de componentes principales con rotación varimax. Se eliminaron los ítems que no cumplieran con el valor mayor a 0.50 de carga Eigen, y se redujo a 43 reactivos de un total de 82, los cuales se distribuyeron en tres áreas (Consideraciones generales, Actividad académicas y Uso de herramientas tecnológicas para la educación) que explicaron $78 \%$ del total de la varianza. Es importante señalar que no se colocan todas las tablas por cuestiones de espacio.

La subescala de Consideraciones generales quedó conformada por un total 5 de 14 ítems: equivale $41.6 \%$. Mientras que la subescala de Actividades académicas quedó conformada por un total 15 de 38 ítems: equivale a $39.4 \%$. Por último, la subescala de Uso de herramientas para la educación se conformó con 23 de un total de 30 ítems: equivale a 76.6 $\%$. En esta última no disminuyó significativamente la cantidad de ítems porque la diversidad del uso dependía de área de académica del personal académico universitario (ver tabla 1).

Tabla 1. Análisis factorial, promedio por subescalas

\begin{tabular}{|l|c|c|c|c|}
\hline \multirow{2}{*}{ Promedio subescala } & \multicolumn{4}{|c|}{ Factores } \\
\cline { 2 - 5 } & 0 & 1 & 2 & 3 \\
\hline & \multicolumn{4}{|c|}{$N=36$} \\
\hline Consideraciones generales: 5 ítems & 0.723 & 0.841 & 0.59 & 0.51 \\
\hline Actividades académicas: 15 ítems & 0.595 & 0.679 & 0.653 & 0.575 \\
\hline Uso de herramientas: 10 ítems & 0.528 & 0.678 & 0.666 & 0.636 \\
\hline
\end{tabular}

Fuente: Elaboración propia

La confiabilidad fue calculada a través del alfa de Cronbach para cada subescala y el total de los reactivos fue de $\alpha=0.78$, lo que evidencia una consistencia interna adecuada (Cronbach, 1998). En las subescalas se encontraron valores de alfa de Cronbach aceptables; el más bajo se encontró en la subescala de Uso de herramientas para la educación por la diversificación de áreas de conocimiento (ver tabla 2). 
Tabla 2. Confiabilidad del instrumento en subescalas y total

\begin{tabular}{|c|c|}
\hline Subescalas & $\alpha$ \\
\hline \multicolumn{2}{|c|}{$N=36$} \\
\hline Consideraciones generales: 5 ítems & 0.77 \\
\hline Actividades académicas: 15 ítems & 0.82 \\
\hline Uso de herramientas para la educación: 10 ítems & 0.75 \\
\hline Total del instrumento & 0.78 \\
\hline
\end{tabular}

Fuente: Elaboración propia

Aunado a lo anterior, se recurrió a la prueba de Kolmogorov-Smirnov para determinar la bondad de ajuste de la muestra en relación con la curva normal, y se obtuvieron coeficientes de asimetría adecuados, lo que se confirma con la prueba de $p=0.79$ (ver tabla $3)$.

Tabla 3. Estadísticos descriptivos de los factores

\begin{tabular}{|l|l|l|l|l|l|}
\hline Subescalas & Media (DE) & P25 & P50 & P75 & $\begin{array}{l}\text { Coeficiente } \\
\text { de } \\
\text { asimetría }\end{array}$ \\
\hline $\begin{array}{l}\text { Consideraciones } \\
\text { generales: } 5 \text { ítems }\end{array}$ & $1.49(0.57)$ & 1.26 & 1.83 & 1.33 & 0.63 \\
\hline $\begin{array}{l}\text { Actividades } \\
\text { académicas: } 15 \text { ítems }\end{array}$ & $2.00(0.65)$ & 1.2 & 2 & 1.6 & 0.774 \\
\hline $\begin{array}{l}\text { Uso de herramientas: } 10 \\
\text { ítems }\end{array}$ & $2.21(0.51)$ & 2 & 2.5 & 2.25 & 0.817 \\
\hline
\end{tabular}

DE: Desviación estándar, $\mathrm{P}=$ Percentil.

Fuente: Elaboración propia

Todo el análisis antes expuesto finaliza con la validez total del instrumento, alfa de Cronbach $\alpha=0.87$, y de confiabilidad calculada, alfa de Cronbach $\alpha=0.78$ (Cronbach, 1998).

\section{Discusión}

La elaboración, validación y estandarización del instrumento en Web para la certificación de académicos universitarios en conocimiento y uso TIC y TAC sirve para agilizar los procesos de evaluación del rubro destinado al personal académico. 


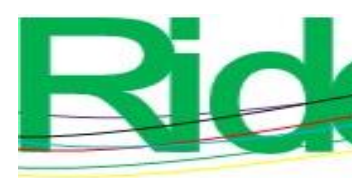

Revista Iberoamericana para la

Investigación y el Desarrollo Educativo

ISSN $2007-7467$

Como se ha expuesto en esta investigación, la evaluación continua de los organismos certificadores es una necesidad en las instituciones de educación superior. Dichos organismos evalúan indicadores y estándares para emitir un dictamen de acreditación de la calidad educativa.

El instrumento para la evaluación es de uso ágil, veraz y confiable. Es de utilidad para definir en qué nivel de conocimiento y uso tienen los académicos en TIC y TAC, de acuerdo con las normas estipuladas por el Copaes y varios expertos en la materia. Los resultados pueden ser editados rápidamente por estar en una página web.

La extensión del instrumento es moderada, cuenta con tan solo 43 ítems, y está dividido en tres subescala (Consideraciones generales, Actividades académicas y Uso de herramientas para la educación) que miden los tres principales factores indispensables y representantes de los tres ejes teóricos (Prendes et al., 2010; Durán, 2015; Durán et al., 2016).

Presenta una validez total de $\alpha=0.87$ y de confiabilidad calculada de $\alpha=0.78$, probado en una población con una distribución normal con $p=0.79$, lo cual muestra aceptable transferencia por ser estandarizado, y permite que el instrumento se utilice en diversas poblaciones de personal académico universitario, como es requerido por el manual de procedimientos editado por el Copaes (2018).

La URL específica del estudio mostró lo puesto a continuación: 1) Nivel muy bajo, con puntaje $\leq 27$ : son académicos que poseen pocos conocimientos y han efectuado poca práctica de las TIC y TAC, por lo que requieren ser capacitados con cursos básicos para que empleen dentro de su materia dichas herramientas tecnológicas. 2) Nivel básico, con puntaje de 28 a 43: académicos que tienen conocimientos, pero usan tan solo eventualmente las TIC y TAC en la práctica porque no han descubierto los beneficios del uso de estas herramientas tecnológicas. Lo que requieren son cursos de motivación que muestren los beneficios de la aplicabilidad de la tecnología en la educación. 3) Nivel medio, con puntaje de 44 a 60: son académicos que tienen conocimientos del tema en cuestión y lo ponen en práctica frecuentemente, lo que les permite ver las ventajas del uso de las herramientas tecnológicas. Esto impacta en sus estudiantes para que sigan utilizando la tecnología en su día a día. 4) Nivel alto, con puntaje $\geq 61$ : docentes que conocen y usan con frecuencia la tecnología en sus cursos, lo que propicia un mejor aprendizaje por parte de los alumnos y los contagia en el uso diario de dichas herramientas, tanto en su vida personal como su vida laboral, como lo demuestran en sus estudios transversales educativos Durán et al. (2016).

El nivel muy bajo y nivel básico requieren de capacitación en TIC y TAC, mientras que el nivel medio y alto puede acceder a promociones de categorización, porque ellos están 


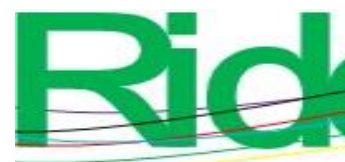

Revista Iberoamericana para la

Investigación y el Desarrollo Educativo

ISSN $2007-7467$

enseñando de forma más eficaz a sus estudiantes y están impactando en estos respecto al buen uso de la tecnología (Cano, 2008). Lo anterior ya está evaluado por los investigadores Moreno y Trigo en 2017, quienes comprueban que la capacitación en TIC y TAC genera en los académicos actitudes positivas dentro de las prácticas y eleva las capacidades de los estudiantes para el aprendizaje, vida y preparación para la vida laboral.

Las certificaciones garantizan la calidad educativa. Los académicos entrenados y calificados de forma deseable con conocimientos educativos digitales, como se requieren hoy en día (Moya, 2013), favorecen la educación del futuro e incrementan los conocimientos digitales en sus estudiantes, lo que posibilita la formación de profesionistas acorde a las demandas de organizaciones mundiales.

\section{Conclusiones}

El instrumento sistematizado presentado en esta investigación es ágil, veraz y confiable. Lo anterior está demostrado desde su construcción porque pasa por varios filtros de validez tanto internos como externos.

La transferencia es aceptable por estar estandarizado en población de docentes universitarios. Lo que permite evaluar al personal docente que requieren ser certificados en niveles de conocimiento y uso de las TIC y TAC.

La aplicabilidad de la certificación es diversa: 1) Selección y contratación de personal, 2) Identificar el nivel conocimientos que tiene el personal, 3) Crear programas y/o cursos que refuerce o cree nuevas habilidades en TIC y TAC, 4) Categorizar al personal de acuerdo su nivel de conocimientos, 5) Promocionar al personal para adquirir beneficios o prestaciones laborales, 6) Evidenciar el nivel que tiene los académicos en la evaluación que realizan los organismos acreditadores y 7) Constancia curricular de posesión de nivel de conocimiento y uso en TIC y TAC en área académica.

Otra de las bondades del instrumento es que puede ser aplicado de forma individual o grupal y está disponible tanto en versión en papel como en modalidad digital (solicitar la página web a los autores). 


\section{Referencias}

Beneitone, P., Esquetini, C., González, J., Maletá, M. M., Siufi, G. y Wagenaar, R. (2007). Reflexiones y perspectivas de la Educación Superior en América Latina. Informe Final - Proyecto TUNING - América Latina 2004-2007. Bilbao, España: Universidad de Deusto.

http://www.sg.inter.edu/uploads/UIPRSG/documentos/asuntos_academicos/RevisionP EG/Proyecto\%20Tuning\%20America\%20Latina_Informe\%20FinalEspanol_2007.pdf.

Cabero, J. y Gutiérrez, J. J. (2015) La producción de materiales TIC como desarrollo de las competencias del estudiante universitario. Aula de Encuentro, 17(2), 5-32. Recuperado de https://revistaselectronicas.ujaen.es/index.php/ADE/article/view/2656/2126.

Cano, M. (2008). La evaluación por competencias en la educación superior. Profesorado. Revista currículum y formación del profesorado, 3(12), 1-16. Recuperado de https://www.ugr.es/ recfpro/rev123COL1.pdf.

Consejo de Acreditación de la Enseñanza en Contaduría y Administración [Caceca]. (2018). Instrumento armonizado para evaluación de reacreditación-licenciatura. Ciudad de México, México: Consejo de Acreditación de la Enseñanza en Ciencias Administrativas Contables y Afines.

Consejo Nacional de Acreditación en Informática y Computación [Conaic]. (2017). Marco de referencia para la Acreditación de Programas Académicos de Informática y Computación. Educación Superior-Énfasis Internacional y Resultado. Ciudad de México, México: Consejo Nacional de Académicos de Informática y Computación. Recuperado de https://www.conaic.net/publicaciones/marco\%20de\%20referencia\%20CONAIC\%20E S\%20y\%20TSU\%202016.pdf.

Consejo Nacional para la Enseñanza e Investigación en Psicología [CNEIP]. (2018a). Manual para la acreditación de programas de Licenciatura en psicología. Ciudad de México, México: Consejo Nacional para la Enseñanza e Investigación en Psicología.

Consejo Nacional para la Enseñanza e Investigación en Psicología [CNEIP]. (2018b). Marco de referencia. Ciudad de México, México: Consejo Nacional para la Enseñanza e $\begin{array}{llll}\text { Investigación en } & \text { Psicología. }\end{array}$ https://www.ugr.es/ recfpro/rev123COL1.pdf.

Consejo para la Acreditación de la Educación Superior [Copaes]. (2016). Marco General de Referencia para los Procesos de Acreditación de Programas Académicos de Tipo Superior. (Ver 3.0). Ciudad de México, México: Consejo para la Acreditación de la 


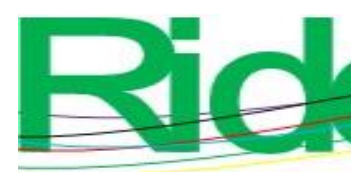

Revista Iberoamericana para la

Investigación y el Desarrollo Educativo

ISSN $2007-7467$

Educación Superior. Recuperado de https://www.copaes.org/assets/docs/Marco-deReferencia-V-3.0_.pdf.

Consejo para la Acreditación de la Educación Superior [Copaes]. (2018). Manual del procedimiento para el reconocimiento de organismos acreditadores de programas académicos de nivel superior. Ciudad de México, México: Consejo para la Acreditación de la Educación Superior. Recuperado de http://sistemasenlinea.uag.mx/Academia/Academia/catalogoOAIES/2_Manual_proced imientos\%20Copaes.pdf.

Cronbach, L. (1998). Fundamentos de los tests psicológicos. España: Biblioteca Nueva.

Durán, C. (2015). Instrumento de Evaluación para la Certificación de la Competencia TIC del Profesorado Universitario. (tesis del programa oficial de Máster y Doctorado en Tecnología Educativa: E-Learning y Gestión del Conocimiento). Universidad de Murcia, España. Recuperado de https://digitum.um.es/xmlui/handle/10201/45536.

Durán, M., Gutiérrez, I. y Prendes, M. (2016). Certificación de la competencia Tic del profesorado universitario. Diseño y validación de un instrumento. Consejo Mexicano de Investigación Educativa, 21(69), 527-556.

Ferrari, A. (2013). DIGCOMP: A framework for developing and understanding digital competence in Europe. Spain: Joint Research Centre. Institute for Prospective Technological Studies.

Retrieved from http://publications.jrc.ec.europa.eu/repository/bitstream/JRC83167/lb-na-26035enn.pdf.

González, E. y Suárez, E. (2016). Adaptación, validación y estandarización de cuestionarios para egresados y empleadores, Estado de México. Revista Iberoamericana para la Investigación y Desarrollo Educativo, 12(6). Recuperado de http://www.ride.org.mx/index.php/RIDE/article/view/222/1003.

González, J. y Wagenaar, R. (2003). TUNING Educational Structures in Europe II. Bilbao, España: Universidad de Deusto. Recuperado de http://tuningacademy.org/wpcontent/uploads/2014/02/TuningEUI_Final-Report_SP.pdf.

Gutiérrez, I. (2011). Competencias del profesorado universitario en relación al uso de tecnologías de la información y comunicación: Análisis de la situación en España y propuesta de un modelo de formación. (tesis doctoral). Universitat Rovira i Virgili, Cataluña, España. Recuperado de https://www.tdx.cat/handle/10803/52835. 


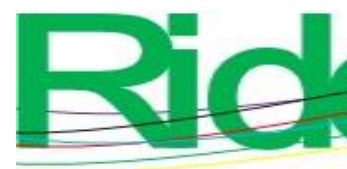

Revista Iberoamericana para la

Investigación y el Desarrollo Educativo ISSN $2007-7467$

Gutiérrez, I. (2014). Perfil del profesor universitario español en torno a las competencias en tecnologías de la información y la comunicación. Pixel-Bit Revista de Medios y Educación, 44, 51-65. Recuperado de https://idus.us.es/xmlui/handle/11441/45804.

Instituto Nacional de Estadística y Geografía [Inegi]. (2012). Clasificación mexicana de programas de estudio por campos de formación académica 2011, educación superior y media superior. Aguascalientes, México: Instituto Nacional de Estadística y Geografía. Recuperado de https://www.copaes.org/assets/docs/Anexo-AClasificacion-Mexicana-de-Programas-de-Estudio.pdf.

Joint Research Center [JRC]. (2017). Evaluar la competencia digital docente, Marco Europeo para la competencia digital del profesorado. (DigCompEdu). Sevilla, España: Joint Research Center, Comisión Europea. Recuperado de https://ec.europa.eu/jrc/sites/jrcsh/files/digcompedu_leaflet_es-nov2017pdf.pdf.

Kerlinger, N. (2009). Investigación del Comportamiento. México: McGraw-Hill.

Llobel, J. P., Pérez, J. F. y Navarro, M. D. (1996). Diseños y la investigación experimental en psicología (2. ${ }^{\mathrm{a}}$ ed.). Valencia, España: Cristóbal Serrano Villalba (CSV).

Moreno, V. P. y Trigo, I. E. (2017). Las TIC y las TAC al servicio de la educación: Una introducción a los mapas conceptuales y la toma de apuntes. Revista de Estudios, 5, 89-103. Recuperado de http://dx.doi.org/10.25267/Rev_estud_socioeducativos.2017.i5.09.

Moya, M. (2013). De las TICs a las TACs: la importancia de crear contenidos educativos digitales. Revista científica de opinión y divulgación, 27, 1-15. Recuperado de http://dim.pangea.org/revistaDIM27/docs/AR27contenidosdigitalesmonicamoya.pdf.

Organización de Cooperación de Desarrollo Económico [OCDE]. (2017). Building an Inclusive Mexico: Policies and Good Governance for Gender Equality. Paris, France: Organization for Economic Cooperation and Development. Retrieved from http://dx.doi.org/10.1787/9789264265493-en.

Organización de Cooperación de Desarrollo Económico [OCDE]. (2018). Education policy outlook, Mexico. Mexico: Organization for Economic Cooperation and Development. Retrieved from http://www.oecd.org/education/Education-Policy-Outlook-CountryProfile-Mexico-2018.pdf.

Organización de las Naciones Unidas para la Educación, la Ciencia y la Cultura [Unesco]. (2016). Declaración de Incheon y Marco de Acción ODS 4 - Educación 2030. Biblioteca digital. República de Corea: Organización de las Naciones Unidas para la 


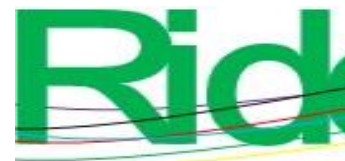

Educación

la

Ciencia

Investigación y el Desarrollo Educativo ISSN $2007-7467$

https://www.gcedclearinghouse.org/sites/default/files/resources/245656s.pdf.

Prendes, M. P. (2010). Competencias TIC para la docencia en la Universidad Pública Española: Indicadores y propuestas para la definición de buenas prácticas: Programa de Estudio y Análisis. Informe del Proyecto EA2009- 0133 de la Secretaría del Estado de Universidades e Investigación. Murcia, España: Universidad de Murcia. Recuperado de https://www.um.es/competenciastic/.

Prendes, M. P., Castañeda, L. y Gutiérrez, I. (2010). ICT Competences of Future Teachers. [Competencias para el uso de TIC de los futuros maestros]. Revista Comunicar, 35, 175-182. Recuperado de https://doi.org/10.3916/C35-2010-03-11.

Prendes, M. P. y Gutiérrez, I. (2013). Competencias tecnológicas del profesorado en las universidades españolas. Revista de Educación, 361, 196-222. Recuperado de http://www.revistaeducacion.mec.es/doi/361_140.pdf.

Ramírez, A. y Casillas, M. A. (Comps.) (2014). Háblame de TIC: Tecnología Digital en Educación Superior. Córdoba, Argentina: Editorial Brujas.

Taquez, H., Rengifo, D. y Mejía, D. (2017). Diseño de un instrumento para evaluar el nivel de uso y apropiación de las TIC en una institución de educación superior. Cali, Colombia: Universidad Icesi. Recuperado de http://recursos.portaleducoas.org/sites/default/files/5030.pdf.

Universidad Autónoma del Estado de México [UAEM]. (2019). Legislación Universitaria. Reglamento de Estudios Profesionales, artículo 04,193. Toluca, México: Universidad Autónoma del Estado de México. Recuperado de https://es.scribd.com/document/254142888/Reglamento-Estudios-Profesionales-. 


\begin{tabular}{|c|c|}
\hline Conceptualización & Elvira Ivone González Jaimes \\
\hline Metodología & Elvira Ivone González Jaimes \\
\hline Software & $\begin{array}{l}\text { Asdrúbal López Chau, } \mathbf{8 0 \%} \\
\text { Valentín Trujillo Mora, 20\% }\end{array}$ \\
\hline Validación & $\begin{array}{l}\text { Asdrúbal López Chau, } \mathbf{8 0 \%} \\
\text { Valentín Trujillo Mora, } 20 \%\end{array}$ \\
\hline Análisis Formal & Jorge Bautista López \\
\hline Investigación & $\begin{array}{l}\text { Elvira Ivone González Jaimes, } \mathbf{7 0 \%} \\
\text { Valentín Trujillo Mora, 30\% }\end{array}$ \\
\hline Recursos & $\begin{array}{l}\text { Elvira Ivone González Jaimes, } 60 \% \\
\text { Asdrúbal López Chau, } 40 \%\end{array}$ \\
\hline Curación de datos & $\begin{array}{l}\text { Elvira Ivone González Jaimes, } 40 \% \\
\text { Asdrúbal López Chau, } 60 \%\end{array}$ \\
\hline $\begin{array}{l}\text { Escritura - Preparación del } \\
\text { borrador original }\end{array}$ & $\begin{array}{l}\text { Valentín Trujillo Mora, 50\% } \\
\text { Jorge Bautista López, 50\% }\end{array}$ \\
\hline Escritura - Revisión y edición & Elvira Ivone González Jaimes \\
\hline Visualización & Elvira Ivone González Jaimes \\
\hline Supervisión & Elvira Ivone González Jaimes \\
\hline Administración de Proyectos & Jorge Bautista López \\
\hline Adquisición de fondos & Universidad Autónoma del Estado de México \\
\hline
\end{tabular}

\section{Anexo 1}

\section{Manual para usuario de la versión en papel}

El instrumento cuenta con 43 ítems. Se dividió en tres subescalas: 1) Consideraciones generales (cinco reactivos), donde se mide la importancia del uso de la tecnología en la educación; 2) Actividades académicas (15 reactivos), donde se mide el uso de la tecnología en el aula para resolver problemas académicos, y 3) Uso de herramientas para la educación (23 reactivos).

Cada ítem presenta una escala del 0 a 3, donde 0 significa 'nunca', 1 significa 'pocas veces', 2 significa 'muchas veces' y 3 significaba 'siempre', para diferenciar la frecuencia de conocimiento y uso de las TIC y TAC.

La evaluación implica los siguientes pasos: 1) Se realiza la sumatoria cada uno de los puntos de cada columna. 2) A la sumatoria se multiplica por $0,1,2$ y 3 , número ya estipulado en cada columna. 3) Se suman todas las columnas multiplicadas para formar el gran total. 4) 
Se busca el rango al que pertenece el gran total para convertirlo al nivel de certificación (ver tabla de conversión del gran total).

Es importante señalar que las partes uno y dos se consideran todos los ítems, pero en la parte tres se considera solo 10 ítems como mínima puntuación, porque va a depender del área de conocimiento del académico. La evaluación se realiza por rangos categorizando a los tres niveles de certificación de la siguiente tabla

Tabla de conversión del gran total

\begin{tabular}{|l|l|}
\hline Rangos del gran total & Nivel de certificación \\
\hline Menor o igual a $(\leq) 27$ & Nivel muy bajo \\
\hline 28 a 43 & Nivel básico \\
\hline 44 a 60 & Nivel medio \\
\hline Mayor o igual a $(\geq) 61$ & Nivel alto \\
\hline
\end{tabular}

\section{Instrumento certificador (versión en papel)}

Instrumento para la certificación de docentes universitarios en conocimiento y uso en tecnologías de la información y la comunicación (TIC) y tecnologías del aprendizaje y el conocimiento (TAC)

Nombre del docente:

Área o departamento académico donde imparte clase:

Edad:

Antigüedad

como

docente:

A continuación, se encuentra una serie de afirmaciones respecto al uso de herramientas tecnológicas para educación. Instrucciones: Marca con una cruz la casilla que corresponda a tu respuesta. Recuerda ser lo más honesto posible.

\section{Parte 1: Consideraciones generales}




\begin{tabular}{|c|c|c|c|c|c|}
\hline \multicolumn{2}{|c|}{$\begin{array}{l}\text { Afirmaciones } \\
\end{array}$} & \multirow[t]{2}{*}{ Nunca } & \multirow{2}{*}{$\begin{array}{l}\text { Pocas } \\
\text { veces }\end{array}$} & \multirow{2}{*}{$\begin{array}{l}\text { Muchas } \\
\text { veces }\end{array}$} & \multirow[t]{2}{*}{ Siempre } \\
\hline 1 & $\begin{array}{l}\text { La educación superior es favorecida por } \\
\text { la implementación y uso de las } \\
\text { tecnologías de la información y } \\
\text { comunicación (TIC) y tecnologías del } \\
\text { aprendizaje y el conocimiento (TAC). }\end{array}$ & & & & \\
\hline 2 & $\begin{array}{l}\text { La política educativa de la institución } \\
\text { donde laboro debería de favorecer la } \\
\text { capacitación y el uso de las TIC y TAC. }\end{array}$ & & & & \\
\hline 3 & $\begin{array}{l}\text { El uso de las TIC es necesario para el } \\
\text { futuro profesional de los estudiantes. }\end{array}$ & & & & \\
\hline 4 & $\begin{array}{l}\text { El uso de las TAC brinda soluciones a } \\
\text { las necesidades de enseñanza. }\end{array}$ & & & & \\
\hline 5 & $\begin{array}{l}\text { Las TAC facilitan el aprendizaje de mis } \\
\text { estudiantes. }\end{array}$ & & & & \\
\hline \multicolumn{6}{|c|}{ Parte 2: Actividad académicas } \\
\hline & Afirmaciones & Nunca & $\begin{array}{l}\text { Pocas } \\
\text { veces }\end{array}$ & $\begin{array}{c}\text { Muchas } \\
\text { veces }\end{array}$ & Siempre \\
\hline 1 & $\begin{array}{l}\text { Utilizo los recursos o herramientas de las } \\
\text { TIC. }\end{array}$ & & & & \\
\hline 2 & $\begin{array}{l}\text { Utilizo las TAC en diversas de } \\
\text { metodologías didácticas. }\end{array}$ & & & & \\
\hline 3 & $\begin{array}{l}\text { Utilizo las TIC y/o TAC para } \\
\text { evaluaciones y autoevaluaciones } \\
\text { educativas. }\end{array}$ & & & & \\
\hline 4 & $\begin{array}{l}\text { Estimulo la participación en TIC y TAC } \\
\text { a mis estudiantes. }\end{array}$ & & & & \\
\hline 5 & $\begin{array}{l}\text { Difundo en las TAC la información } \\
\text { didáctica autogenerada. }\end{array}$ & & & & \\
\hline 6 & $\begin{array}{l}\text { Aprendo a usar herramientas y/o } \\
\text { aplicaciones TIC y TAC de forma } \\
\text { autónoma. }\end{array}$ & & & & \\
\hline
\end{tabular}




\begin{tabular}{|c|c|c|c|c|c|}
\hline 7 & $\begin{array}{l}\text { Soluciono problemas académicos } \\
\text { utilizando las TAC. }\end{array}$ & & & & \\
\hline 8 & $\begin{array}{l}\text { Reporto la escasez de equipamiento o } \\
\text { fallas técnicas presentadas en mi clase. }\end{array}$ & & & & \\
\hline 9 & $\begin{array}{l}\text { Trabajo en equipo con actividades de } \\
\text { TAC académicas. }\end{array}$ & & & & \\
\hline 10 & $\begin{array}{l}\text { Intercambio con otros docentes mis } \\
\text { reflexiones, experiencias y recursos } \\
\text { sobre el uso de las TIC y/o TAC. }\end{array}$ & & & & \\
\hline 11 & $\begin{array}{l}\text { Promuevo el uso de las TIC y/o TAC } \\
\text { fuera del aula. }\end{array}$ & & & & \\
\hline 12 & $\begin{array}{l}\text { Planeo mis clases en función de las TAC } \\
\text { a usar. }\end{array}$ & & & & \\
\hline 13 & $\begin{array}{l}\text { Pruebo la utilidad de las TIC y TAC } \\
\text { antes de usarlas en clase. }\end{array}$ & & & & \\
\hline 14 & $\begin{array}{l}\text { Brindo asesorías sobre TIC y/o TAC } \\
\text { fuera de la clase. }\end{array}$ & & & & \\
\hline 15 & $\begin{array}{l}\text { Promuevo autoaprendizaje en TAC para } \\
\text { los estudiantes. }\end{array}$ & & & & \\
\hline \multicolumn{6}{|c|}{ Paste 3: Uso de herramientas tecnológicas para la educación } \\
\hline \multicolumn{2}{|r|}{$\begin{array}{c}\text { Frecuencias de uso de herramientas para } \\
\text { educación }\end{array}$} & Nunca & $\begin{array}{l}\text { Pocas } \\
\text { veces }\end{array}$ & $\begin{array}{c}\text { Muchas } \\
\text { veces }\end{array}$ & Siempre \\
\hline 1 & Correo electrónico (Gmail, Yahoo, etc.). & & & & \\
\hline 2 & $\begin{array}{l}\text { Foros de discusión (dentro de alguna } \\
\text { plataforma o algún sitio de Internet). }\end{array}$ & & & & \\
\hline 3 & $\begin{array}{l}\text { Servicio de mensajería (Whatsapp, } \\
\text { Telegram, Facebook, etc.). }\end{array}$ & & & & \\
\hline 4 & $\begin{array}{l}\text { Videoconferencia (Skype, Hangouts, } \\
\text { Zoom, etc.). }\end{array}$ & & & & \\
\hline 5 & $\begin{array}{l}\text { Redes sociales o redes sociales visuales } \\
\text { (Facebook, Twitter, Instagram, } \\
\text { Instagram, Pinterest, etc.). }\end{array}$ & & & & \\
\hline 6 & Redes profesionales (Linkedin,, Xing, & & & & \\
\hline
\end{tabular}




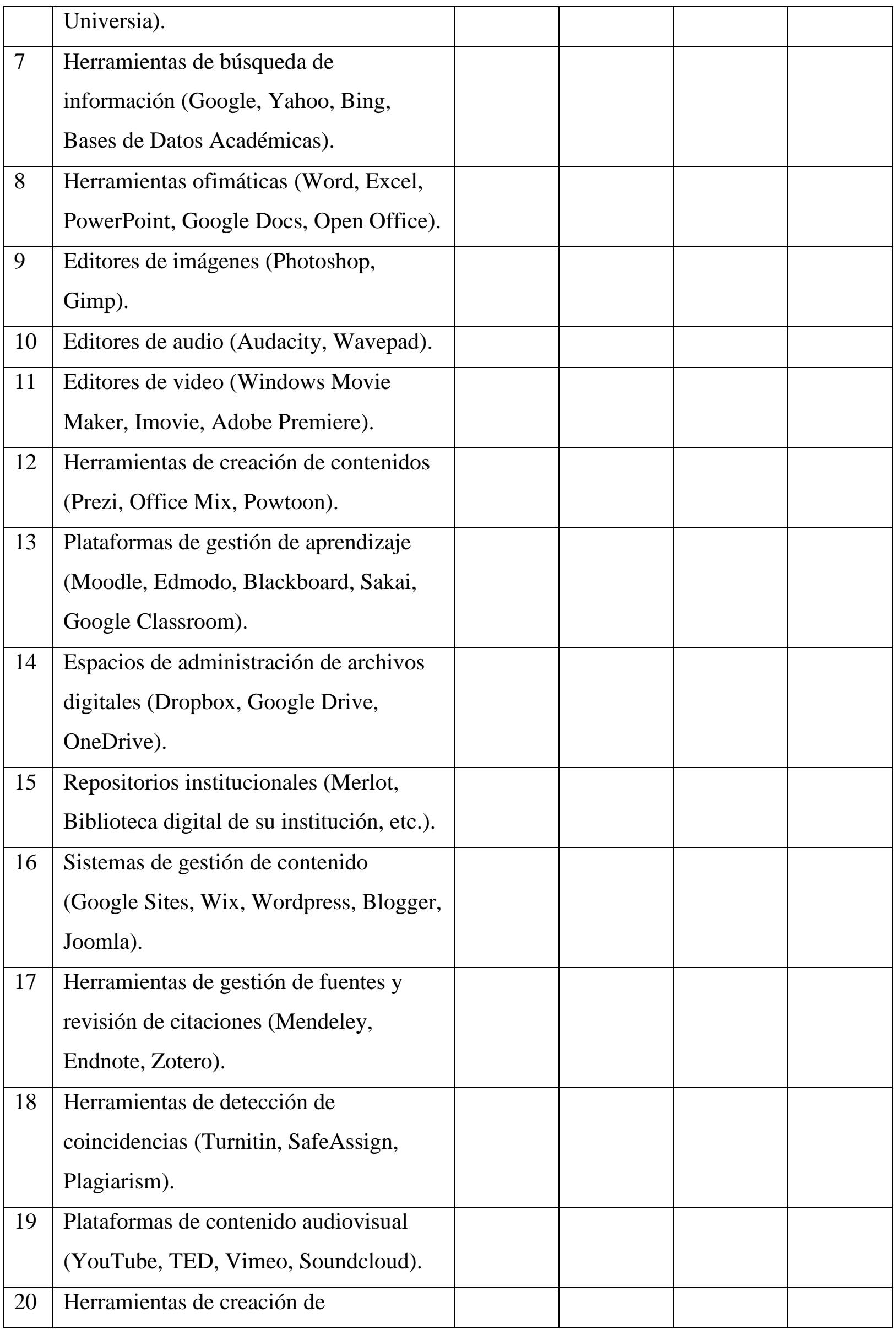




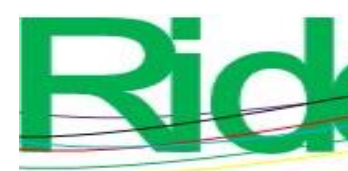

Revista Iberoamericana para la Investigación y el Desarrollo Educativo ISSN 2007-7467

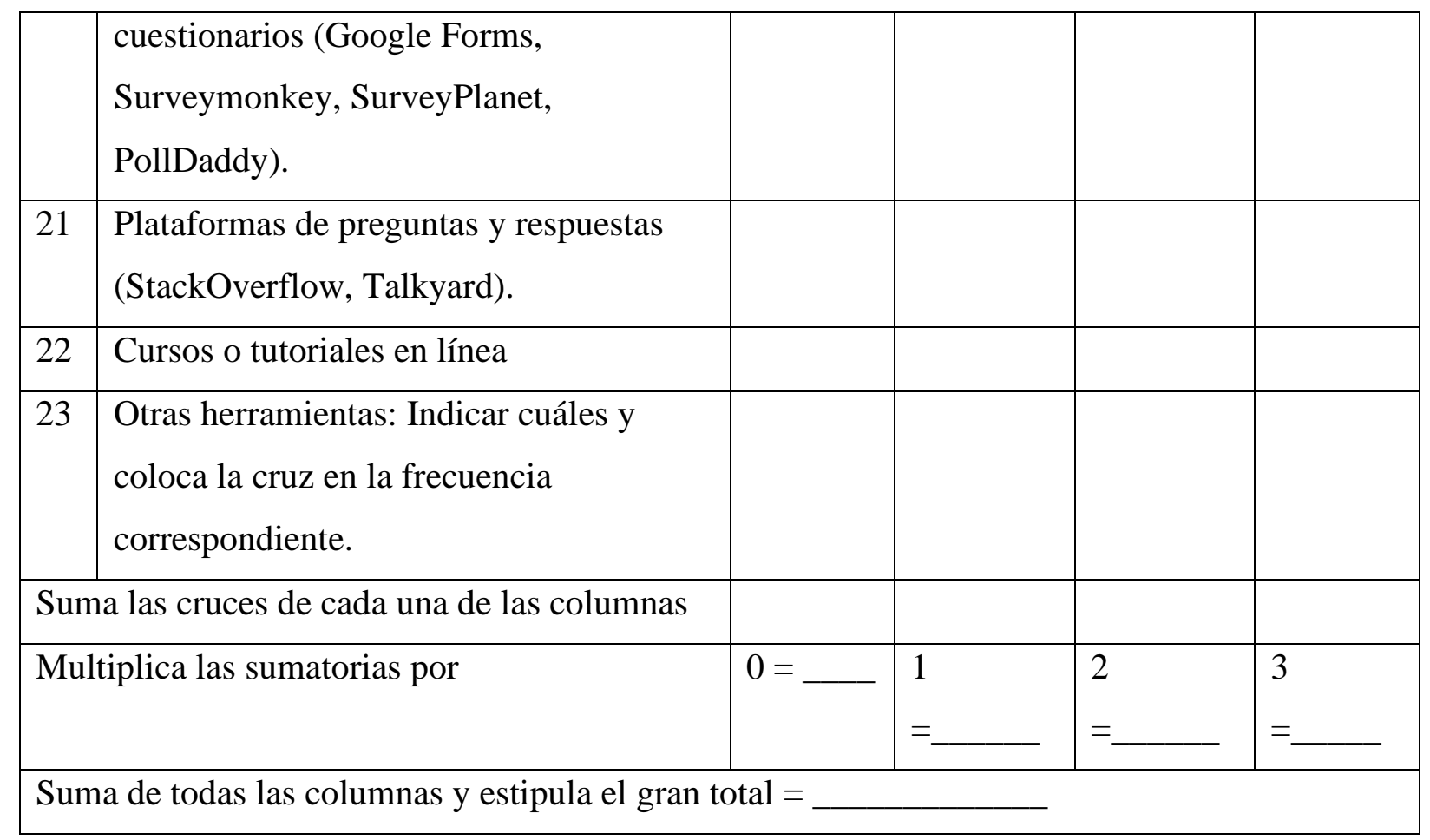

